Volume 1 (Issue 1) (2017) Pages 30-34

\title{
Integration and Remote Monitoring of Wireless Sensor Networks in the Development of SMART Villages
}

\author{
Ray Mulenga ${ }^{1}$, Dr. Josephat Kalezhi ${ }^{2}$ Sonile K Musonda ${ }^{3}$ \\ ${ }^{1,3}$ Department of Electrical Engineering, School of Engineering, Copperbelt University, Kitwe, Zambia \\ ${ }^{2}$ Department of Computer Science, SMNS, Copperbelt University, Kitwe, Zambia \\ raymulenga@gmail.com ${ }^{1}$,Kalezhi@cbu.ac.zm², sonile@cbu.ac.zm³
}

\begin{abstract}
In Zambia there are 10 provinces of which 8 are essentially in rural areas where more than $80 \%$ of the population live (1). Successive Zambian governments have made several attempts at increasing budgetary allocation towards improving rural agriculture particularly in the advent of climate change (2). This paper discusses an automated drip irrigation system employing ZigBee technology. In many applications where high-end equipment is installed, lack of maintenance support for remote villages means systems often fall into disrepair and eventual vandalism (3). With the rapid expansion of the Internet, there is the added potential for the remote control and monitoring of such systems. The paper discusses the designing and building of irrigation systems for villages which are independent, efficient and self-sustaining in providing smart services and solutions particularly in developing agro industries.
\end{abstract}

Keywords-smart village, rural area, wireless sensor networks (WSN) and precision agriculture.

\section{INTRODUCTION}

The IEEE Smart villages initiative defines a "Smart Village" as an off-grid community that integrates solar energy for the creation of locally owned entrepreneurial businesses and generation of affordable all whether agro businesses (4). The concept of Smart villages is a global modern approach for offgrid communities.

\section{A. Literature Review}

To realize the novelty concept of smart village a whole bouquet of technologies will have to be deployed in order to cover the entire ecosystem of life at Mwinilunga village. The system described by Dlodlo and Kalezhi [5] relates to creating a network of objects that communicate with one another via the internet, integrating with embedded sensors, Radio Frequency Identifier (RFID), General Packet Radio Service (GPRS), computers, mobile phones e.t.c. These objects exchange and process information according to defined tasks and send reports to users.

Most of the literature in academia on smart villages lies predominantly along solving the energy question and leaves it up to the community to extrapolate as to what applications would be relevant to them. Such a system is what rural electrification authority (REA) in Zambia has implemented in some parts of the country since 2003. My argument is that it doesn't create a clearly defined relationship between energy access and sustainable livelihood in terms of agriculture. Secondly it doesn't incorporate a telemetry system which facilitates remote monitoring and management.

An automated system done by Manimaran (6), has two main units. One is wireless sensor unit (WSU) and another is Wireless information unit (WIU). Wireless sensor unit transmits the sensor data to the wireless information unit. Wireless information unit receives sensor data from wireless sensors. Each unit is based on the micro-controller that controls the radio modem ZigBee and processes information from the soil-moisture sensor, temperature sensor and water level sensor. In this wireless sensor unit or transmission unit the sensor data from different sensors (Soil moisture, temperature, humidity and water level) are collected in the main controller. This data is displayed on transmission section liquid crystal display. A controller is programmed to some threshold values of temperature and soil moisture. Sensed values are compared with the threshold values and according to comparison automation takes place.

Kumar et. al (7) discussed a plant watering system aimed at developing a mechanism to conserve water and reduce errors due to human negligence. It uses moisture sensors to sense the level of moisture in the soil. When the moisture content of the soil goes below a certain limit for a plant/crop, the pump system is triggered and the plant/crop is watered. The plants are watered efficiently till the desired value is reached and the pump is switched off automatically.

\section{B. Features of the proposed System}

Resident at the core of this design is a proposed solar plant with sufficient capacity to meet the energy needs of the community of Mwinilunga village. This system uses soil moisture, temperature, voltage, water level and current sensors. These sensors send values to the Atmega328P microcontroller via a wireless sensor network using the ZigBee protocol. It creates a distributed wireless network of soil moisture and temperature sensors placed in the root zone of the plants, water level sensor is placed in tank for checking the water level in tank. The voltage and current sensors are placed 
in the solar energy plant to determine operating conditions. The micro-controller handles all the sensor information, triggers actuators, and transmits data to a web application for remote monitoring [8].

The system is designed to be flexible and scalable, allowing additional sensors to be easily and safely added to the network with the minimum amount of effort. In the interest of sustainability, the system will allow agricultural extension officers to monitor and control connected devices in the village through a gateway running on the Global System for Mobile (GSM)/GPRS module. I have decided to use GSM because in Mwinilunga only second-generation of cellular telephone technology $(2 \mathrm{G})$ services are available. $2 \mathrm{G}$ network was the first to use digital encryption of conversations and the first to offer data services and SMS text messaging, but their data transfer rates are lower than those of their successors like Universal Mobile Telecommunication System (UMTS) or 3G (9). A gateway is implemented to facilitate interoperability between heterogeneous networks and provide a consistent interface, regardless of the accessing device (9). This paper is organized as follows: Section 2 discusses the problem statement and research objectives. Section 3. Discusses the methodology and prototype design. Section 4 describes the implementation of the proposed system 5. provides a conclusion.

\section{CASE STUDY}

The people of Mwinilunga village of North-Western province of Zambia fall into the category of the "total poor" (1) and therefore fit the profile of a case study to examine the possibility of deploying a Smart Village initiative. These rural areas face a number of challenges in the domains of agriculture, environmental management, finance, communications infrastructure, connectivity, water resources management, sanitation, roads and transport, access to markets, health and education, which calls for locally relevant solutions to be directed towards solving the question of sustainability (5). The scope of this research is firstly, to identify agricultural needs of Mwinilunga village in the light of an ever changing climatic environment. Secondly, in response to these needs, design a prototype drip irrigation system which will contribute to lessening the impact of drought conditions in Zambian villages. Thirdly, I plan to design a tailor made telemetry system which will provide remote monitoring by the agricultural extension officers.

My research endeavors to ask the following questions:

(1) What are the energy needs of the rural communities such that, when addressed, will lead to the establishment of a smart village?

(2) Is automation in agriculture really useful and in what part or parts of the cultivation process (e.g. seed planting, growing, harvesting, selling) can it be applied?
(3) What is the cost of the cultivation process and how can this cost be reduced by automating one or more parts of this process?

The following objectives will therefore be considered:

(1) Review existing literature on the agricultural challenges in Mwinilunga village.

(2) Review literature related to wireless sensor networks.

(3) Design and construction of an automated irrigation system with telemetry capability.

\section{METHODOLOGY}

The approach adopted is to investigate the previously implemented systems and to find the most suitable technologies that can be applied to focus the research and to build a suitable and valuable system (10). The study will examine wireless sensor network architectures and applications in the agricultural sector utilizing a qualitative method (11). This method will give us a better understanding of why and how the process should be designed. More specifically, the work can be split into the following parts:

(1) Literature study and field survey,

(2) Design of a prototype solution and

(3) Implementation of the prototype

The literature study provides the background information that is necessary for understanding the feasibility of the design and the previously implemented solutions.

\section{SYSTEM DESIGN AND MPLEMENTATION}

Mwinilunga village is located in the north-western province of Zambia some $872 \mathrm{Km}$ from the capital Lusaka. It is an isolated community of over 1000 people living off the national grid (13). It survives purely on agriculture, with the cassava being the main cash crop grown. It has a primary school and a clinic or health center. To realize the objectives, we have proposed the system depicted in figure 1, which provides a telemetry system allowing a remote station with the ability to manage site information. It further permits the field technicians to interact with the instrumented environment remotely in-terms of system performance and maintenance. The system proposed consists of wireless sensor nodes which collects soil moisture, control irrigation rate and monitor energy consumption from the solar plant. Figure 3 depicts such a system using an ATmega328P micro-controller as the processing unit, and an array of sensors interconnected in a wireless mesh network using the ZigBee nodes. 


\section{A. Hardware design}

1. Wireless Sensor Network

Wireless sensor networks (WSN), sometimes called wireless sensor and actuator networks (WSAN) are spatially distributed autonomous sensors to monitor physical or environmental conditions, such as temperature, sound, pressure, etc. and to cooperatively pass their data through the network to a main location (13). The WSN is built of "nodes" from a few to several hundreds or even thousands, where each node is connected to one or several sensors. Each such sensor network node has typically several parts: a radio transceiver with an internal antenna or connection to an external antenna, a microcontroller, an electronic circuit for interfacing with the sensors and an energy source, usually a battery or an embedded form of energy harvesting.

\section{ZigBee Protocol}

ZigBee is an IEEE 802.15.4-based standard which specifies a low-cost, low-power, wireless mesh network for a suite of high-level communication protocols (13). It is suitable for operation in isolated locations.

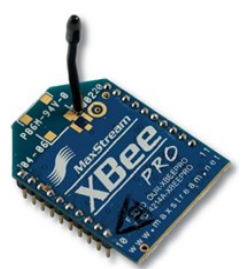

Figure 2: ZigBee Module from Digi International Inc. (19)

The ZigBee standard supports three device types: ZigBee Coordinator, ZigBee Router, and ZigBee End Device (14). The IEEE 802.15.4 standard defines the physical and MAC (Medium Access Control) layers for low-rate wireless personal area networks. The physical layer supports three frequency bands with different gross data rates: $2,450 \mathrm{MHz}$ (250 kbs-1), $915 \mathrm{MHz}$ (40 kbs-1) and $868 \mathrm{MHz}$ (20 kbs-1).

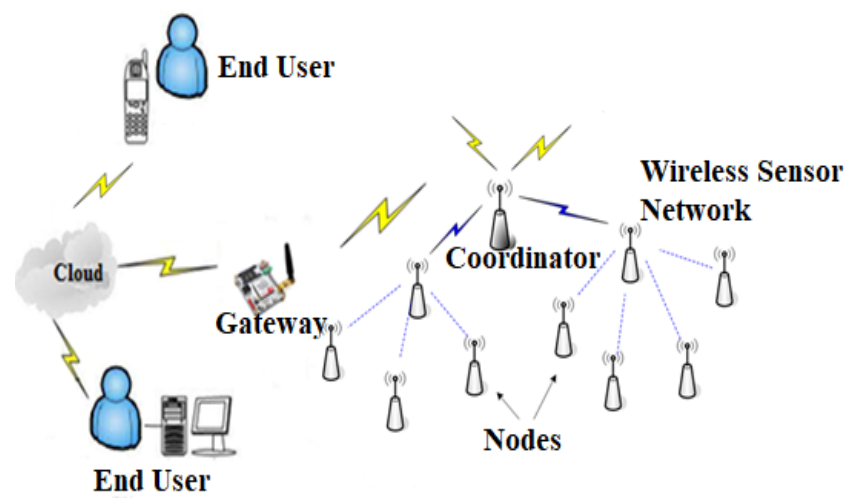

Figure 3: ZigBee based wireless sensor network

\section{Telemetry System}

Chavan and Karande (13) discussed a method of remote control and monitoring systems based on GSM-ZigBee remote control and monitoring system with automatic irrigation. The design was running on ZigBee protocol. This system will apply a soil moisture, water level, voltage, current and temperature sensors to connect to a micro-controller through a wireless sensor network established by the ZigBee nodes as depicted in figure 4.
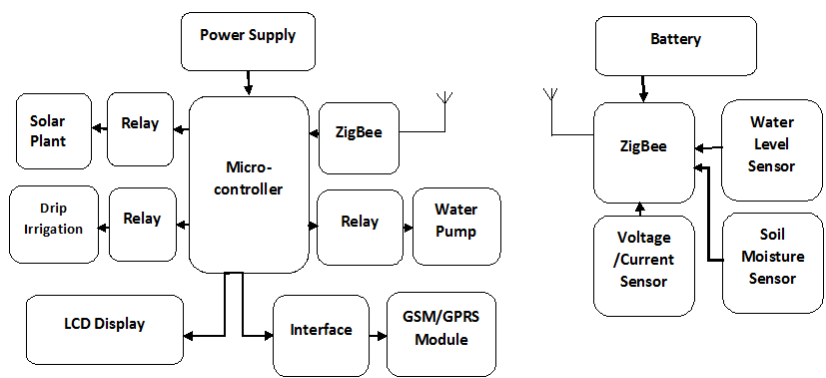

Figure 4: Block diagram

Figure 5 is a design circuit of an automatic irrigation and telemetry system using the Arduino Uno micro-controller development board. It uses a Soil Hygrometer moisture-sensor to read the moisture content of the soil. The GSM module SIM900D is used for telemetry, while the XBee nodes form the core of the wireless sensor network (WSN) to which multiple sensors from the monitored objects are connected. A $16 \times 4$ LCD is connected with Atmega328P in 4-bit mode. The LM041L is the LCD module used here. LM041L is a $16 \times 4$ LCD module with 16 pins and can be operated in 4-bit mode

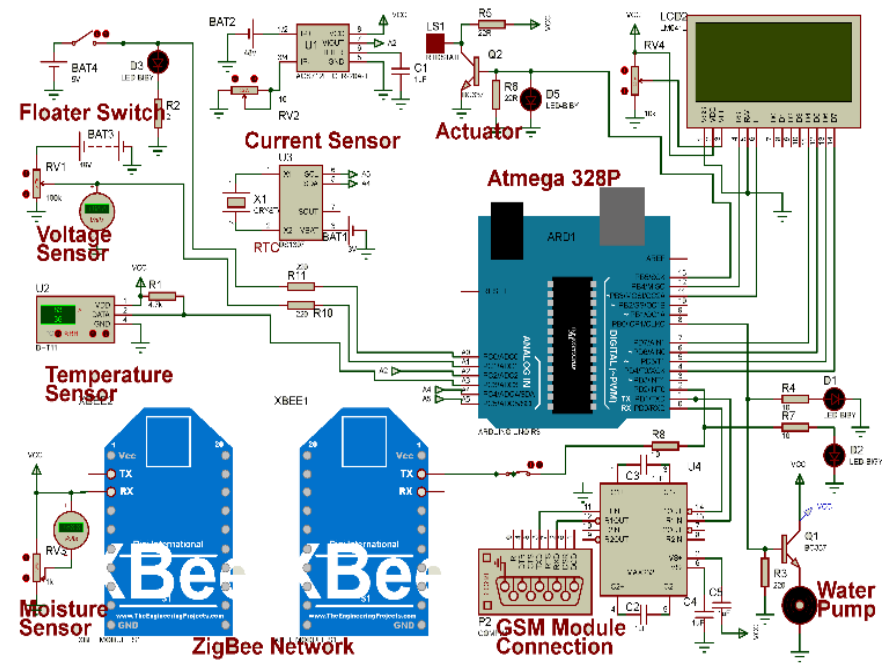

Figure 5: Circuit design 


\section{Simulation Results}

Using the virtual serial ports software, we were able to create a virtual bridge between port 1 of XBee 1 and port 2 of XBee 2 in order to simulate a wireless connection in proteus software. The Arduino uno Rev3 development board is built around the Atmega328P micro-controller. In its simplicity, the circuit switches on the water pump, when the floater switch is closed, symbolizing that the water tank is full and vice-versa. The Voltage sensor together with the current sensor (ACS712), provide the parameters to determine the power consumption and state of health of the solar plant. The DS1309 provides the real time clock (RTC). The XBee 1 provides a high output if the moisture sensor input is high and vice-versa. This causes the processor to operate the actuator which either opens the irrigation pipes or closes them depending upon the amount of moisture content in the soil. The proteus library does not have a default GSM SIM900D modem library. I had to download the closest GSM library for proteus software from the engineering projects blog (20) as depicted in figure 6. It does support the basic AT commands of the SIM900D module.

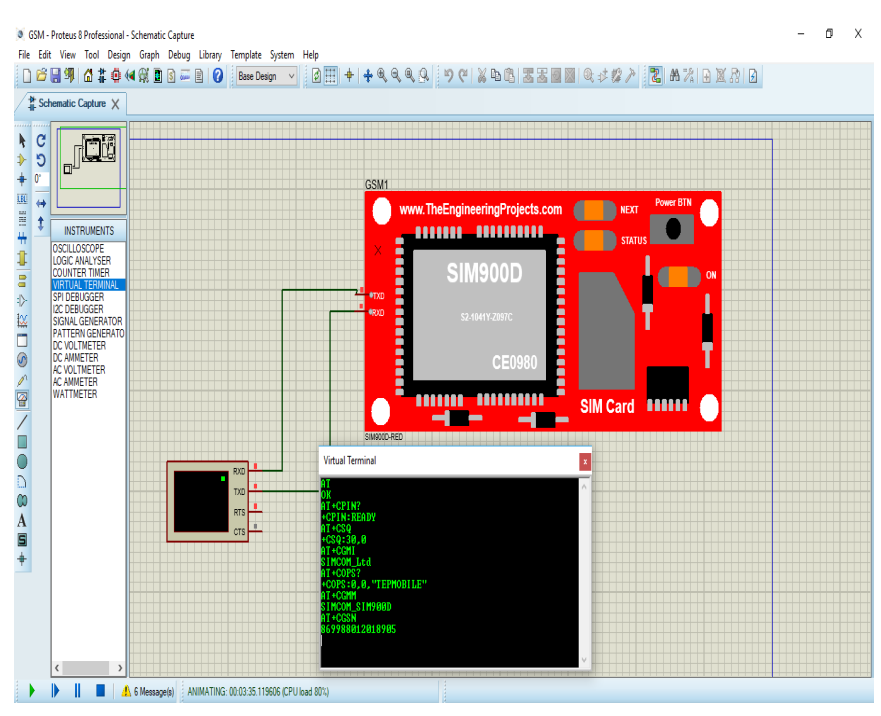

Figure 6: Simulation output for SIM900D modem

\section{IMPACT ON CROP YIELD AND SUSTAINABILITY}

Instead of making decisions based in some hypothetical average condition, which may not exist anywhere in reality, a precision farming approach recognizes differences and adjusts management actions accordingly (15). Projections indicate that from 1999 to 2030 irrigated land will increase by $33 \%$ (16). The application of wireless sensor network (WSN) for a water irrigation, control and monitoring is composed of a number of networking sensor nodes that can be deployed to detect the places where the water level is low. The system will then deploy actuators to open drip irrigation to that particular area only (17). This will allow us to conserve water and minimize wastage. This method of crop irrigation is increasingly being referred to as "Precision irrigation" and is an important practice in water-saving agriculture cropping system, which allows producers to maximize their productivity while saving water. Rural villages will be empowered with the latest information regarding weather data from the meteorological department which will help them in planning for the season as well as communicating with agricultural experts. This will be accomplished through the inbuilt telemetry system within the design.

\section{CONCLUSION}

Zambia has a current urbanization rate of $3.2 \%$ per year leading to overcrowding in cities, pollution, destruction of agricultural land and parks including outbreak of contagious disease. Consequently, decreasing urbanization in the long run has numerous benefits to the community (18). To mitigate this, it's important to invigorate underdeveloped areas by making agriculture more productive and sustainable and increasing the resilience of livelihoods to threats and crises.

\section{Reference}

[1] George Lwanda and Paul Quarles van Ufford, "Addressing the multiple dimensions of Poverty and Inequality in Zambia" UN Zambia Signature Issues Serie-1 (UN), [Online] Available: www.zm.one.un.org/download/file/fid/223 p.5 June 2013.

[2] Ministry of Agriculture and Co-operatives, "National Agricultural Policy (2012 - 2030)" [Online] Available: http://www.g-fras.org/fr/2015-05-28-15-50-27/australia22.html?download=319:the-national-agriculture-policy2012-2030.

[3] Samanvay, "SmartVillages: A Community Driven Initiative Reconstructing Rural India" [Online] Available: www.smartvillages .org, 2012.

[4] IEEE Smart Village; an IEEE Foundation Signature Program, [Online] Available: www.ieefoundation.org/smart-village.

[5] Nomusa Dlolo and Josephat Kalezhi, "The Internet of Things in Agriculture for Sustainable Rural Development" International Conference on Emerging Trends in Networks and Computer Communications. pp 13-18 2015.

[6] P. Manimaran et.al, "An Intelligent Smart Irrigation System Using WSN and GPRS Module" International Journal of Applied En gineering Research ISSN 0973-4562 Volume 11, Number 6 (2016) pp 3987-3992.

[7] Nirdosh Kumar et.al, "Smart Farming System for Indian Farmers using Arduino based Technology" International Journal of Advance Research, Ideas and Innovations in Technology, (Volume3, Issue1, 2017). [Online] Available: www.ijariit.com.

[8] Khusvinder Gill, et. al "A ZigBee-based Home Automation system" IEEE Transactions on Consumer Electronics, vol. 55, No. 2. pp 422-430 (2009). 
[9] GSMARENA "Network coverage in Zambia" [Online] Available: http://www.gsmarena.com/network -bands.php3?s Country=Zambia (2017).

[10] IEEE Smart Village, "Integrating sustainable electricity, education, and entrepreneurial solutions to empower off-grid communities" ieee-smart-village.org, 2016.

[11] Wireless Sensor-based Agricultural Monitoring System.

[12] Emmanuel Kamwithe. et. Al, Civil society for poverty reduction, "Mwinilunga district poverty monitoring report" $\mathrm{p}$. 18,2003

[13] Wikipedia encyclopedia, "Wireless sensor networks" [Online] Available: en.wikipedia.org, 2016.

[14] Chavan and Karande, "Wireless Monitoring of Soil Moisture, Temperature \& Humidity Using Zigbee in Agriculture",

[15] Karandeep Kaur, "The idea of Smart villages based on Internet of Things (IoT)", International Research Journal of Engineering and Technology (IRJET) e-ISSN: 2395 -0056 Volume: 03 Issue: 05 | May-2016.

[16] Maria Culman et. al, "A review of Wireless Sensor Networks for crop field monitoring and considerations for its application in Colombian agriculture" Second International Congress of Mechanical Engineering and Agricultural Science, 2015.

[17] C. H. Chavan, and P. V.Karande, "Wireless Monitoring of Soil Moisture, Temperature \& Humidity Using Zigbee in Agriculture" International Journal of Engineering Trends and Technology (IJETT) - Volume pp. 493-497,2014.

[18] Elizabeth Brown, "How to Reduce Urbanization", [Online] Available: www.peopleofoureverydaylife.com, 2016

[19] Digi XBee/rf solutions and accessories. [Online] Available: https://www.digi.com/products/xbee-rf-solutions 